\title{
Latitudinal and longitudinal dependence of the cosmic ray diurnal anisotropy during 2001-2014
}

\author{
Anastasia Tezari ${ }^{1, *}$, Helen Mavromichalaki ${ }^{1}$, Dimitrios Katsinis ${ }^{1}$, Anastasios Kanellakopoulos ${ }^{1}$, Sofia Kolovi ${ }^{1}$, \\ Christina Plainaki ${ }^{2,1}$, and Maria Andriopoulou ${ }^{3}$ \\ ${ }^{1}$ Nuclear and Particle Physics Department, Faculty of Physics, National and Kapodistrian University of Athens, \\ Zografos, 15784 Athens, Greece \\ ${ }^{2}$ INAF-IAPS, Via del Fosso del Cavaliere, 00133, Rome, Italy \\ ${ }^{3}$ Space Research Institute, Austrian Academy of Sciences, Graz, Austria \\ * Invited contribution by A. Tezari, recipient of the EGU Outstanding Student Poster (OSP) Award 2015.
}

Correspondence to: Helen Mavromichalaki (emavromi@phys.uoa.gr)

Received: 4 July 2016 - Revised: 14 October 2016 - Accepted: 18 October 2016 - Published: 21 November 2016

\begin{abstract}
The diurnal anisotropy of cosmic ray intensity for the time period 2001 to 2014 is studied, covering the maximum and the descending phase of solar cycle 23, the minimum between solar cycles 23 and 24, and the ascending phase and maximum of solar cycle 24. Cosmic ray intensity data from 11 neutron monitor stations located at different places around the Northern Hemisphere obtained from the high-resolution Neutron Monitor Database (NMDB) were used. Special software was developed for the calculations of the amplitude and the phase of the diurnal anisotropy vectors on annual and monthly basis using Fourier analysis and for the creation of the harmonic dial diagrams. The geomagnetic bending for each station was taken into account in our calculations determined from the asymptotic cones of each station via the Tsyganenko96 (Tsyganenko and Stern, 1996) magnetospheric model. From our analysis, it was resulted that there is a different behavior of the diurnal anisotropy vectors during the different phases of the solar cycles depending on the solar magnetic field polarity. The latitudinal and longitudinal distribution of the cosmic ray diurnal anisotropy was also examined by grouping the stations according to their geographic coordinates, and it was shown that diurnal variation is modulated not only by the latitude but also by the longitude of the stations. The diurnal anisotropy during strong events of solar and/or cosmic ray activity is discussed.
\end{abstract}

Keywords. Interplanetary physics (cosmic rays)

\section{Introduction}

The spatial anisotropy of the galactic cosmic radiation (GCR) in the interplanetary medium is observed as the daily variation in cosmic ray (CR) intensity which is recorded by ground-based detectors. A detector on Earth scans the entire sky during a time period of $24 \mathrm{~h}$, since the Earth completes one rotation around its own axis once in this time range (Singh et al., 2013). Consequently, the detectors scan through different portions of the $\mathrm{CR}$ angular distribution with a 1-day period. The projection of this anisotropy on the ecliptic plane may be observed as diurnal anisotropy (Yeeram and Saengdokmai, 2015). As a result, the intensity of GCR recorded by ground-based neutron monitors (NMs) shows periodic and abrupt changes as a function of space, time, and energy (Oh et al., 2010). This phenomenon, which is known as the diurnal anisotropy of CR intensity, is a localtime short-term variation (Pomerantz and Duggal, 1971; Ahluwalia, 1988).

The diurnal variation is due to complex phenomena, deriving from the convective-diffusive theory, which involves the radial convection of GCR flux by the solar wind and the inward diffusion along the interplanetary magnetic field (IMF) (Parker, 1964; Rao, 1972; Forman and Gleeson, 1975; Sabbah, 2013). An energy-independent anisotropic flow of CR particles in the $18 \mathrm{~h}$ co-rotational direction is generated due to the equilibrium between the convection and diffusion mechanisms (Krymsky, 1964; Rao, 1972; Mishra and Mishra, 2008). This can explain the long-term average, but 


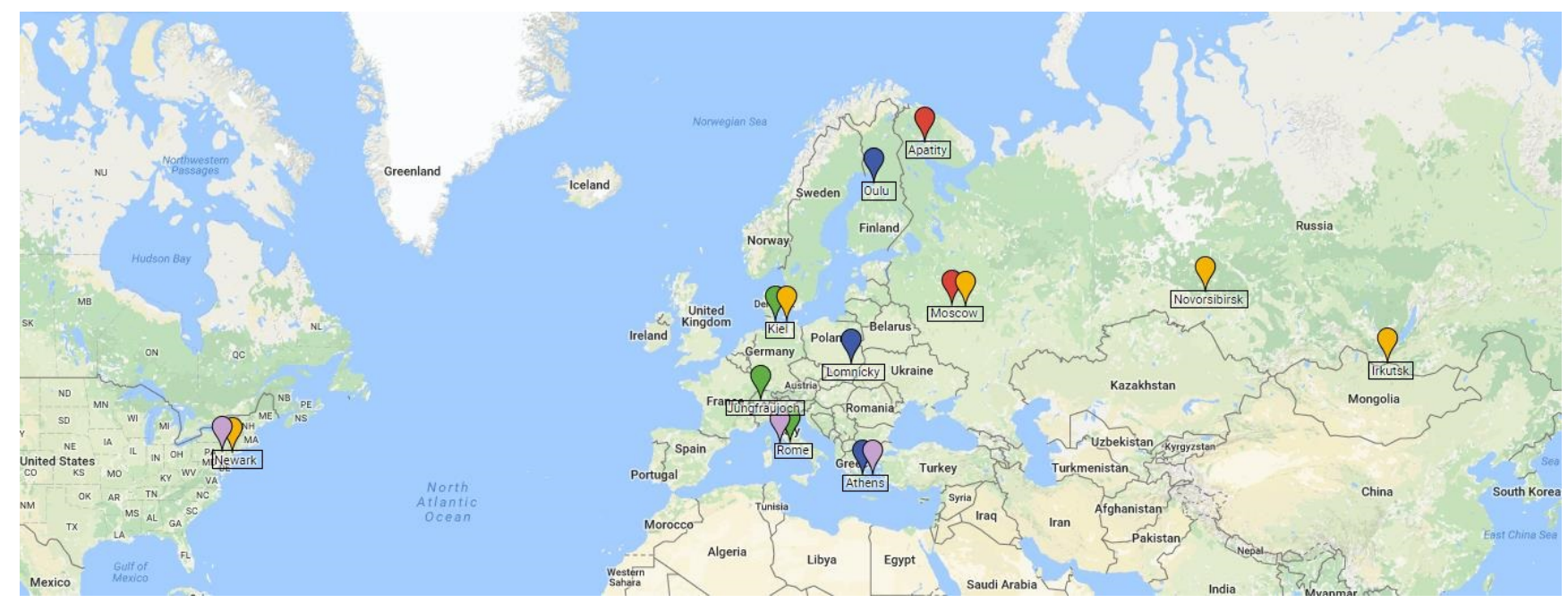

Figure 1. A map giving the geographic locations of the NM stations used in this work. The grouped stations are indicated by the same color.

not short-term variations in diurnal anisotropy (Yeeram and Saengdokmai, 2015). The diurnal anisotropy is also modulated by the geographic coordinates and the altitude of the detectors' location on Earth (Mailyan and Chilingarian, 2010).

The diurnal amplitude follows the 11-year variation in the solar cycle (SC) (Bieber and Chen, 1991; Tiwari et al., 2012), while the diurnal phase probably displays a correlation with the magnetic solar cycle (22-year variation). This is due to the reversal of the solar magnetic field (SMF) around solar maximum activity (Ahluwalia, 1988). Consequently, a significant variability of the diurnal anisotropy vector is observed in terms of amplitude and time of maximum, when considered on a SC variation basis (Mishra and Mishra, 2005). The average diurnal amplitude was calculated on the order of $0.6 \%$ but sometimes can be as great as $1.5 \%$ (Forman and Gleeson, 1975). The continually changing conditions in the interplanetary space cause a large day-to-day variability in the solar diurnal variation in $\mathrm{CR}$ intensity. Phenomena related to solar and CR variations may also be affecting the diurnal anisotropy. Such phenomena include groundlevel enhancements (GLEs), Forbush decreases (FDs) and magnetospheric effects (MEs), which are not interpreted in the same way by every NM (Burlaga and Ness, 1998; Plainaki et al., 2007, 2014). The characteristics of diurnal anisotropy show a remarkable variation during these extreme events (Tezari and Mavromichalaki, 2016).

In this work the diurnal anisotropy of the CR intensity recorded at selected NM stations of the worldwide neutron monitor network, located in the Northern Hemisphere, with different geographic coordinates and threshold rigidity is studied. The stations are grouped according to their geographic latitudes and longitudes, taking into account their asymptotic cones of viewing. The amplitude and the time of maximum of the $\mathrm{CR}$ diurnal anisotropy vectors during the different phases of SCs 23 and 24 and during intense CR events are examined and discussed.

\section{Data analysis}

Values of the CR intensity recorded at the NM stations of Apatity (APTY), Athens (ATHN), Jungfraujoch (JUNG), Irkutsk (IRKT), Kiel (KIEL), Lomnický štít (LMKS), Moscow (MOSC), Newark (NWRK), Novosibirsk (NVBK), Oulu (OULU), and Rome (ROME) that are hourly corrected for pressure and efficiency have been used in this work. These data are obtained from the high-resolution Neutron Monitor Database (NMDB; http://www.nmdb.eu) or from the websites of each individual station. A list of these stations with their characteristics such as the geographic coordinates, the altitude, the cut-off rigidity, and the geomagnetic bending are given in Table 1 . These stations are widely distributed around the Northern Hemisphere and are separated into five groups in order to study the latitudinal distribution of the CR diurnal anisotropy (same geographic longitude), as well as the longitudinal one (same geographic latitude). The location of these NMs is indicated in the map of Fig. 1, with the different colors corresponding to the different groups. These groups are GR 1 (shown in blue), including the stations ATHN, LMKS, and OULU; GR 2 (green), including the stations JUNG, KIEL, and ROME; GR 3 (red), including the stations APTY and MOSC; GR 4 (orange), including the stations IRKT, KIEL, MOSC, NWRK, and NVBK; and GR 5 (purple), including the stations ATHN, ROME, and NWRK. The latitudinal distribution of CR intensity is studied using the groups 1, 2, and 3 based on stations with almost the same longitude and different latitude, while the longitudinal distribution with the groups 4 and 5 based on stations with similar latitude and different longitude. 
Table 1. Characteristics of the NM stations used in this work.

\begin{tabular}{llrrrrr}
\hline NMs & Type & Geogr. lat. & Geogr. long. & Alt. (m) & Rc (GV) & Gb (h) \\
\hline Apatity (APTY) & 18NM64 & $67.57^{\circ} \mathrm{N}$ & $33.4^{\circ} \mathrm{E}$ & 181 & 0.65 & 2.16 \\
Oulu (OULU) & 9NM64 & $65.05^{\circ} \mathrm{N}$ & $25.47^{\circ} \mathrm{E}$ & 15 & 0.81 & 2.27 \\
Kiel (KIEL) & 18NM64 & $54.34^{\circ} \mathrm{N}$ & $10.12^{\circ} \mathrm{E}$ & 54 & 2.36 & 3.05 \\
Newark (NWRK) & 9NM64 & $39.68^{\circ} \mathrm{N}$ & $75.75^{\circ} \mathrm{W}$ & 50 & 2.40 & 3.51 \\
Moscow (MOSC) & 24NM64 & $55.47^{\circ} \mathrm{N}$ & $37.32^{\circ} \mathrm{E}$ & 200 & 2.43 & 2.85 \\
Novosibirsk (NVBK) & 24NM64 & $54.48^{\circ} \mathrm{N}$ & $83.00^{\circ} \mathrm{E}$ & 163 & 2.91 & 2.96 \\
Irkutsk (IRKT) & 12NM64 & $52.47^{\circ} \mathrm{N}$ & $104.03^{\circ} \mathrm{E}$ & 2000 & 3.64 & 3.44 \\
Lomnický štít (LMKS) & 8NM64 & $49.20^{\circ} \mathrm{N}$ & $20.22^{\circ} \mathrm{E}$ & 2634 & 3.84 & 3.58 \\
Jungfraujoch (JUNG) & 3NM64 & $46.55^{\circ} \mathrm{N}$ & $7.98^{\circ} \mathrm{E}$ & 3570 & 4.50 & 3.94 \\
Rome (ROME) & 20NM64 & $41.86^{\circ} \mathrm{N}$ & $12.47^{\circ} \mathrm{E}$ & 0 & 6.27 & 4.67 \\
Athens (ATHN) & 6NM64 & $37.58^{\circ} \mathrm{N}$ & $23.47^{\circ} \mathrm{E}$ & 260 & 8.53 & 4.93 \\
\hline
\end{tabular}

In the frame of this work a new Java-based software application, called the DIurnal Anisotropy Suite (DIAS), is prepared by the authors. DIAS enables the study and the calculation of the amplitude and the time of maximum of the diurnal anisotropy vectors of CR for every day and for a large number of days. This tool is capable of processing large data sets with a high level of automation. Various filters can be applied in order to achieve higher data quality and eliminate the effect of certain physical phenomena that are not useful in our analysis. The selected filters used in this work are discussed in the next paragraph and data are imported from .txt files with a special format provided by the users. The filtered data can be presented on a harmonic dial and polar diagrams of monthly, annual, and multiannual diurnal anisotropy vectors can be generated automatically for a single station or group of stations. This allows the comparative study of the short-term and long-term CR diurnal anisotropy. The graphs are generated automatically by Java software. This tool will be soon available online on the web of the Athens Neutron Monitor Station (ANeMoS; http://cosray.phys.uoa.gr) with the appropriate documentation.

The hourly values of the CR intensity, which have been corrected for pressure, have been normalized with respect of the mean value of the year 2001, which is the year of the solar maximum and consequently the year of the cosmic ray minimum, and are presented in Fig. 2. We discard those days that exhibit an absolute value of the relative deviation of the average daily intensity with respect to the year 2001 that is greater than $25 \%$. Furthermore, in order to eliminate major effects that may distort the long-term diurnal anisotropy, such as GLEs and FDs, individual days with a difference between maximum and minimum intensity greater than $5 \%$ of the average daily intensity are also excluded (Bieber and Chen, 1991; Kudela et al., 2008a). Days with gaps of more than $8 \mathrm{~h}$ are not used in this study (Yeeram and Saengdokmai, 2015). The average number of days per year used after the applied filters is approximately 361 for APTY, 351 for ATHN, 219 for IRKT, 361 for JUNG, 339 for KIEL, 346 for LMKS, 359

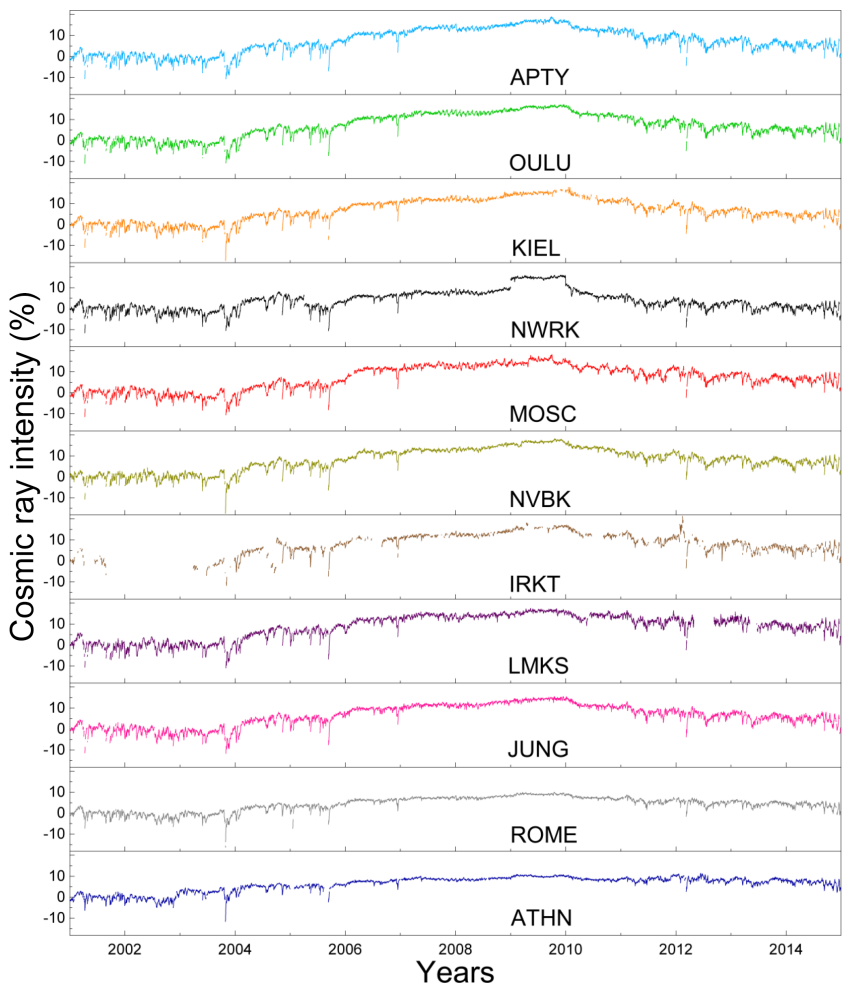

Figure 2. Time profiles of the cosmic ray intensity normalized to the mean value of the period 2001-2014 for all selected stations.

for MOSC, 360 for NWRK, 362 for NVBK, 361 for OULU, and 360 for ROME.

In order to plot the diurnal variation vectors, the diurnal anisotropy characteristics (amplitude and time of maximum) are calculated for each day using Fourier analysis according to the equation

$I_{i}=f\left(t_{i}\right)=I_{\text {mean }}+A\left(\cos \omega t_{i}+\varphi\right)$,

where $I_{\text {mean }}$ represents the daily average of CR intensity, $A$ the amplitude, $\varphi$ the phase of diurnal variation, $\omega=$ $2 \pi / 24\left(\mathrm{~h}^{-1}\right)$ and $i=1,2, \ldots 24$ (Firoz and Kudela, 2007; 
Firoz, 2008). Our data have been normalized according to the equation

$A_{i}=\frac{\left|I_{i}-I_{\text {mean }}\right|}{I_{\text {mean }}} 100(\%)$.

In order to calculate the time of maximum of the diurnal anisotropy in local time (LT), a continuous study of the asymptotic cones of acceptance of the NMs was performed. The cones for all used NMs were calculated using the Tsyganenko96 magnetospheric model (Tsyganenko and Stern, 1996). The asymptotic cones of acceptance for the four groups of NM stations are calculated by using the accurate magnetic field data of 1 January 2003 corresponding to quiet geomagnetic conditions $(\mathrm{Kp}=0$, Dst $=0)$. Then the geomagnetic bending value, $\mathrm{Gb}$, for each $\mathrm{NM}$ was calculated using the asymptotic longitude of each NM (point 1) and calculating the angle between this point and the point presenting the maximum CR flux (point 2) in the eastern direction (Hatton and Carswell, 1963; Shea et al., 1965). An example is given for the Athens NM in Fig. 3a. Thus, the equation for the calculation of the diurnal phase in LT including the geomagnetic bending correction for each station is given by the following relation:

$\mathrm{LT}=\mathrm{UT}+\Delta T+\mathrm{Gb}$,

where LT is the time of maximum in local time, UT is the corresponding one in universal time, $\Delta T$ is the transition time from UT to LT, and Gb is the correction for the geomagnetic bending. More specifically, $\Delta T$ is an integer parameter expressed in hours and gives the difference of the time zones of each station. The above equation can be written for each station as follows:

$$
\begin{aligned}
& \mathrm{LT}_{\mathrm{APTY}}=\mathrm{UT}+3+2.16(\mathrm{~h}) \quad \mathrm{LT}_{\mathrm{NEWK}}=\mathrm{UT}-5+3.51(\mathrm{~h}) \\
& \mathrm{LT}_{\mathrm{OULU}}=\mathrm{UT}+2+2.27(\mathrm{~h}) \quad \mathrm{LT}_{\mathrm{LMKS}}=\mathrm{UT}+1+3.58(\mathrm{~h}) \\
& \mathrm{LT}_{\mathrm{MOSC}}=\mathrm{UT}+3+2.85(\mathrm{~h}) \quad \mathrm{LT}_{\mathrm{JUNG}}=\mathrm{UT}+1+3.94(\mathrm{~h}) \\
& \mathrm{LT}_{\mathrm{NVBK}}=\mathrm{UT}+6+2.96(\mathrm{~h}) \quad \mathrm{LT}_{\mathrm{ROME}}=\mathrm{UT}+1+4.67(\mathrm{~h}) \\
& \mathrm{LT}_{\mathrm{KIEL}}=\mathrm{UT}+1+3.05(\mathrm{~h}) \quad \mathrm{LT}_{\mathrm{ATHN}}=\mathrm{UT}+2+4.93(\mathrm{~h}) \\
& \mathrm{LT}_{\text {IRKT }}=\mathrm{UT}+8+3.44(\mathrm{~h}) \text {. }
\end{aligned}
$$

Using the results of the geomagnetic study, the annual average values of the amplitude and the time of maximum in LT and UT for all the NM stations are calculated and are given in Table 2 (a, b, c). The calculated diurnal vectors of the cosmic ray intensity according to Eqs. (1), (2), (3), and (4) for the selected stations are presented on a harmonic dial on a yearly and monthly basis (Mavromichalaki, 1989; Tezari and Mavromichalaki, 2016; Mavromichalaki et al., 2016) using simple vector calculus and the newly developed DIAS software. The length of the vector represents the diurnal amplitude, while the vector's direction is equivalent to the time of maximum.

\section{Geomagnetic bending}

The asymptotic cone of acceptance of a NM is defined as the solid angle of the asymptotic directions of approach of CR particles of various energies outside the influence of the geomagnetic field and can contribute significantly to the counting rate of the detector (McCracken et al., 1968; Razdan and Summers, 1965; Mishra and Mishra, 2008). The diurnal variation in the $\mathrm{CR}$ intensity at high-latitude stations, where the asymptotic cones of approach scan the meridian, provides a good comprehension of the longitudinal distribution of the anisotropy averaged in time and latitudes according to the asymptotic trajectories (Dorman and Fischer, 1968). The effective cut-off rigidities of the NMs have been taken into account for the calculation of the geomagnetic bending (Storini et al., 1999). The effect of the geomagnetic field on the particle trajectories is of great importance for the study of the diurnal anisotropy of CR intensity as it may affect the diurnal amplitude.

In the current analysis, the asymptotic directions of viewing for the NMs are calculated using the Tsyganenko96 magnetospheric model (Tsyganenko, 1989, 1995; Tsyganenko and Stern, 1996; Belov et al., 2005), which is a semiempirical best-fit representation of the Earth's magnetic field, based on a large satellite observations data sets. Using different field models, especially during geomagnetic disturbances or GLEs (studied in the context of diurnal wave in Sect. 4.3), may lead to different transmissivity of CR (Kudela et al., 2008b; Desorgher et al., 2009).

The term "NM asymptotic cone" is used to define the set of allowed trajectory traces, as expected by the Stormer theory. In the current paper, we perform calculations in order to derive the intersections of the allowed particle trajectories with the atmospheric layer at the altitude of $80 \mathrm{~km}$ (for a description of the applied method see Plainaki et al., 2007, 2009). Since each trajectory corresponds to a different energy (rigidity), we obtain for each NM station a set of allowed positions defined by latitude-longitude pair values. The magnetospheric windows for all NMs used in this work are defined and presented in Fig. 3a, b, c, d, and e. For all the groups of stations, each point refers to the axis of the cone (as it is for particles arriving vertically) and to a particular rigidity. There is a variation step depending on the particle energy; therefore, each point of the diagram from west to east direction corresponds to a different particle rigidity, beginning at $999 \mathrm{GV}$ (which is the energy of a particle detected at the station). From 18.00 to $0.80 \mathrm{GV}$ the step is constant $(0.20 \mathrm{GV})$. It is observed that the asymptotic cones have as a starting point the given NM and unfold with direction towards the Equator. The closer to the Equator a station is, the larger the part of its cone spiraling around the Equator is. This effect offers better and broader view (geographical latitude-wise) of the ecliptic plane due to the high value of magnetic rigidity in such areas. On the other hand, NMs of high geographical 
Table 2. Mean annual values of (a) the normalized diurnal amplitude, (b) the time of maximum in UT, and (c) the time of maximum in LT of cosmic ray intensity recorded at the selected NM stations for the time period 2001-2014.

\begin{tabular}{|c|c|c|c|c|c|c|c|c|c|c|c|}
\hline \multicolumn{12}{|c|}{ (a) Yearly diurnal amplitude (\%) } \\
\hline YEAR & APTY & OULU & KIEL & NWRK & MOSC & NVBK & IRKT & LMKS & JUNG & ROME & ATHN \\
\hline 2001 & 0.84 & 0.90 & 0.82 & 0.95 & 0.81 & 0.91 & 0.86 & 0.85 & 0.93 & 0.79 & 0.89 \\
\hline 2002 & 0.79 & 0.89 & 0.82 & 0.93 & 0.81 & 0.88 & 0.89 & 0.87 & 0.95 & 0.78 & 0.91 \\
\hline 2003 & 0.73 & 0.83 & 0.77 & 0.89 & 0.74 & 0.77 & 0.79 & 0.80 & 0.90 & 0.75 & 0.92 \\
\hline 2004 & 0.71 & 0.80 & 0.75 & 0.87 & 0.75 & 0.76 & 0.80 & 0.80 & 0.84 & 0.72 & 0.86 \\
\hline 2005 & 0.76 & 0.87 & 0.79 & 0.89 & 0.78 & 0.80 & 0.67 & 0.80 & 0.87 & 0.72 & 0.98 \\
\hline 2006 & 0.66 & 0.76 & 0.71 & 0.76 & 0.65 & 0.72 & 0.60 & 0.70 & 0.77 & 0.62 & 0.84 \\
\hline 2007 & 0.62 & 0.69 & 0.66 & 0.72 & 0.60 & 0.66 & 0.54 & 0.65 & 0.72 & 0.58 & 0.79 \\
\hline 2008 & 0.58 & 0.65 & 0.77 & 0.66 & 0.57 & 0.62 & 0.48 & 0.59 & 0.68 & 0.54 & 0.76 \\
\hline 2009 & 0.53 & 0.62 & 0.83 & 0.62 & 0.52 & 0.56 & 0.60 & 0.54 & 0.65 & 0.54 & 0.71 \\
\hline 2010 & 0.63 & 0.75 & 1.43 & 0.72 & 0.64 & 0.66 & 0.71 & 0.66 & 0.75 & 0.59 & 0.82 \\
\hline 2011 & 0.73 & 0.80 & 0.92 & 0.83 & 0.73 & 0.75 & 0.82 & 0.78 & 0.83 & 0.68 & 0.88 \\
\hline 2012 & 0.74 & 0.85 & 0.96 & 0.88 & 0.78 & 0.78 & 0.73 & 0.94 & 0.90 & 0.70 & 1.00 \\
\hline 2013 & 0.72 & 0.81 & 0.90 & 0.85 & 0.71 & 0.73 & 0.69 & 0.84 & 0.83 & 0.65 & 0.87 \\
\hline 2014 & 0.71 & 0.79 & 0.85 & 0.84 & 0.69 & 0.74 & 0.86 & 0.73 & 0.83 & 0.66 & 0.86 \\
\hline \multicolumn{12}{|c|}{ (b) Yearly diurnal phase (UT) } \\
\hline 2001 & 12.30 & 12.97 & 13.14 & 15.72 & 12.45 & 10.55 & 10.57 & 13.26 & 12.81 & 12.45 & 11.00 \\
\hline 2002 & 12.57 & 12.88 & 13.18 & 14.86 & 12.59 & 10.37 & 9.98 & 12.93 & 12.78 & 12.59 & 11.56 \\
\hline 2003 & 12.41 & 13.05 & 13.33 & 15.11 & 12.84 & 9.97 & 9.13 & 13.06 & 12.95 & 12.84 & 12.86 \\
\hline 2004 & 12.45 & 12.89 & 12.81 & 16.17 & 12.36 & 9.04 & 9.00 & 13.07 & 13.10 & 12.36 & 12.36 \\
\hline 2005 & 12.44 & 13.15 & 13.05 & 16.30 & 12.32 & 10.07 & 7.74 & 13.05 & 12.93 & 12.32 & 13.14 \\
\hline 2006 & 12.41 & 12.88 & 13.02 & 16.03 & 12.31 & 9.24 & 7.81 & 12.73 & 12.21 & 12.31 & 11.59 \\
\hline 2007 & 12.07 & 13.20 & 12.94 & 15.95 & 12.24 & 8.91 & 8.14 & 12.60 & 12.74 & 12.24 & 12.44 \\
\hline 2008 & 12.18 & 12.60 & 12.74 & 15.23 & 12.05 & 8.92 & 8.52 & 12.65 & 12.55 & 12.05 & 12.32 \\
\hline 2009 & 12.26 & 12.08 & 12.90 & 14.83 & 11.55 & 8.81 & 8.14 & 12.89 & 12.38 & 11.55 & 12.20 \\
\hline 2010 & 12.47 & 12.49 & 12.54 & 15.24 & 12.35 & 9.83 & 9.80 & 12.92 & 12.66 & 12.35 & 11.82 \\
\hline 2011 & 13.09 & 13.08 & 12.50 & 15.14 & 12.86 & 10.78 & 8.88 & 13.00 & 13.23 & 12.86 & 12.47 \\
\hline 2012 & 12.87 & 12.83 & 12.42 & 15.53 & 12.13 & 10.67 & 8.94 & 12.03 & 12.56 & 12.13 & 12.02 \\
\hline 2013 & 12.63 & 12.51 & 12.17 & 14.88 & 12.59 & 9.71 & 9.22 & 12.53 & 12.92 & 12.59 & 11.55 \\
\hline 2014 & 12.10 & 12.14 & 11.88 & 14.48 & 11.42 & 9.63 & 10.57 & 11.94 & 12.16 & 11.42 & 11.67 \\
\hline \multicolumn{12}{|c|}{ (c) Yearly diurnal phase (LT) } \\
\hline 2001 & 17.46 & 17.24 & 17.19 & 15.23 & 18.14 & 19.51 & 22.01 & 17.84 & 17.75 & 18.12 & 17.93 \\
\hline 2002 & 17.73 & 17.15 & 17.23 & 14.37 & 17.94 & 19.33 & 21.42 & 17.51 & 17.72 & 18.26 & 18.49 \\
\hline 2003 & 17.57 & 17.32 & 17.38 & 14.62 & 17.92 & 18.93 & 20.57 & 17.64 & 17.89 & 18.51 & 19.79 \\
\hline 2004 & 17.61 & 17.16 & 16.86 & 15.68 & 17.79 & 18.00 & 20.44 & 17.65 & 18.04 & 18.03 & 19.29 \\
\hline 2005 & 17.60 & 17.42 & 17.10 & 15.81 & 17.74 & 19.03 & 19.18 & 17.63 & 17.87 & 17.99 & 20.07 \\
\hline 2006 & 17.57 & 17.15 & 17.07 & 15.54 & 17.13 & 18.20 & 19.25 & 17.31 & 17.15 & 17.98 & 18.52 \\
\hline 2007 & 17.23 & 17.47 & 16.99 & 15.46 & 16.99 & 17.87 & 19.58 & 17.18 & 17.68 & 17.91 & 19.37 \\
\hline 2008 & 17.34 & 16.87 & 16.79 & 14.74 & 16.68 & 17.88 & 19.96 & 17.23 & 17.49 & 17.72 & 19.25 \\
\hline 2009 & 17.42 & 16.35 & 16.95 & 14.34 & 16.67 & 17.77 & 19.58 & 17.47 & 17.32 & 17.22 & 19.13 \\
\hline 2010 & 17.63 & 16.76 & 16.59 & 14.75 & 16.92 & 18.79 & 21.24 & 17.50 & 17.60 & 18.02 & 18.75 \\
\hline 2011 & 18.25 & 17.35 & 16.55 & 14.65 & 18.09 & 19.74 & 20.32 & 17.58 & 18.17 & 18.53 & 19.40 \\
\hline 2012 & 18.03 & 17.10 & 16.47 & 15.04 & 17.74 & 19.63 & 20.38 & 16.61 & 17.50 & 17.80 & 18.95 \\
\hline 2013 & 17.79 & 16.78 & 16.22 & 14.39 & 17.79 & 18.67 & 20.66 & 17.11 & 17.86 & 18.26 & 18.48 \\
\hline 2014 & 17.26 & 16.41 & 15.93 & 13.99 & 17.11 & 18.59 & 22.01 & 16.52 & 17.10 & 17.09 & 18.60 \\
\hline
\end{tabular}




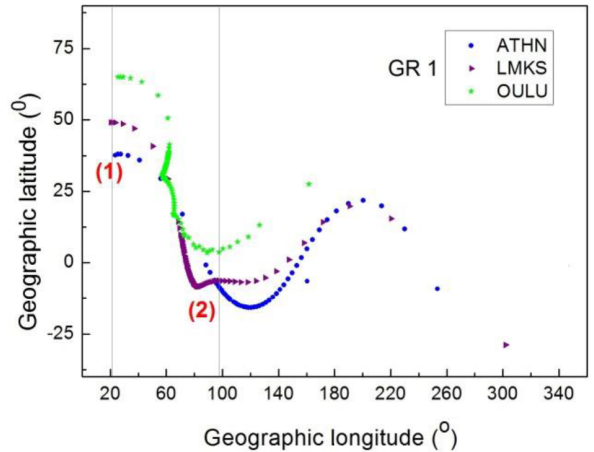

(a)

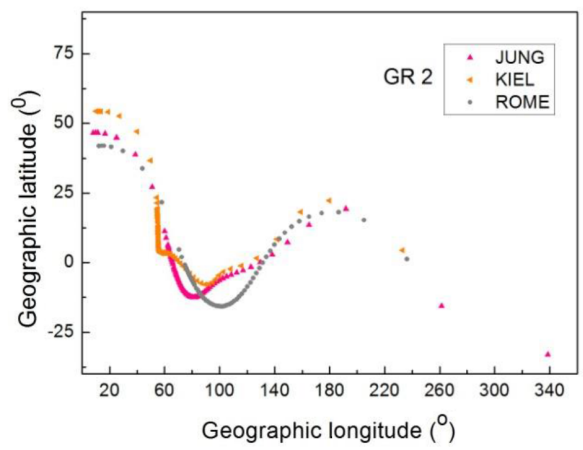

(b)

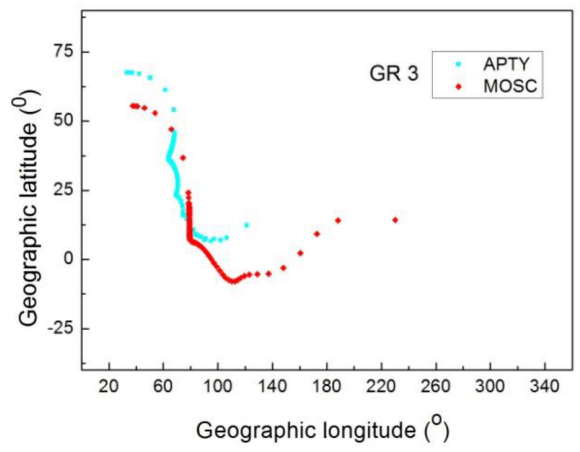

(c)

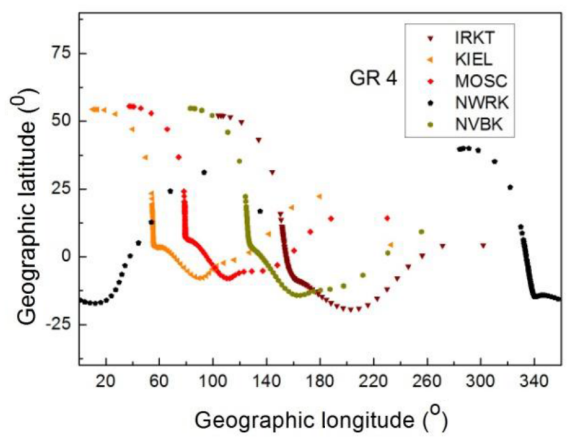

(d)

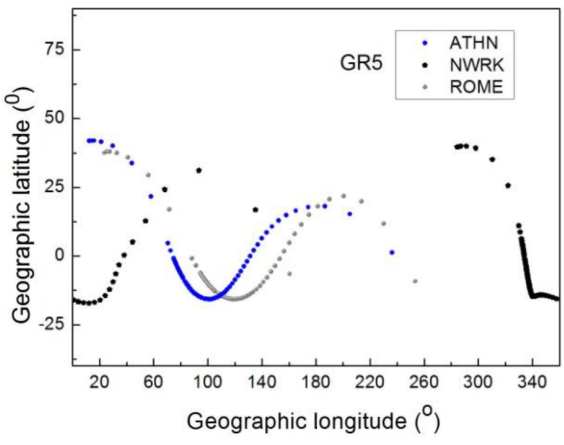

(e)

Figure 3. Asymptotic directions of viewing for all the groups of the NM stations GR 1, GR 2, GR 3, GR 4, and GR 5 obtained by the Tsyganenko96 model corresponding to the quiet geomagnetic period of 1 January 2003.

longitude have a better and a broader view of the area around their meridian all the way to the Equator.

In order to calculate the diurnal anisotropy phase, the geomagnetic bending of each station derived from the above analysis was taken into account in our estimations. It is known that a charged particle moving through a magnetic field is subjected to a Lorentz force, causing a deflection from the particle's original trajectory and magnetic bending, which is a measure of this deflection. In the magnetosphere of the Earth, this force, causing bending of particle tracks, acts on particles of cosmic radiation which consists predominantly of positively charged protons, $\alpha$-particles, and other nuclei (Chaloupka et al., 1970).

It is observed from the Fig. 3 that the asymptotic cones of acceptance of the same group NMs (GR 1, GR 2, GR 3) almost coincide, while the asymptotic cones of the longitudinal groups (GR 4, GR 5) present the same general features with a parallel shift to the east since the Earth's magnetic 


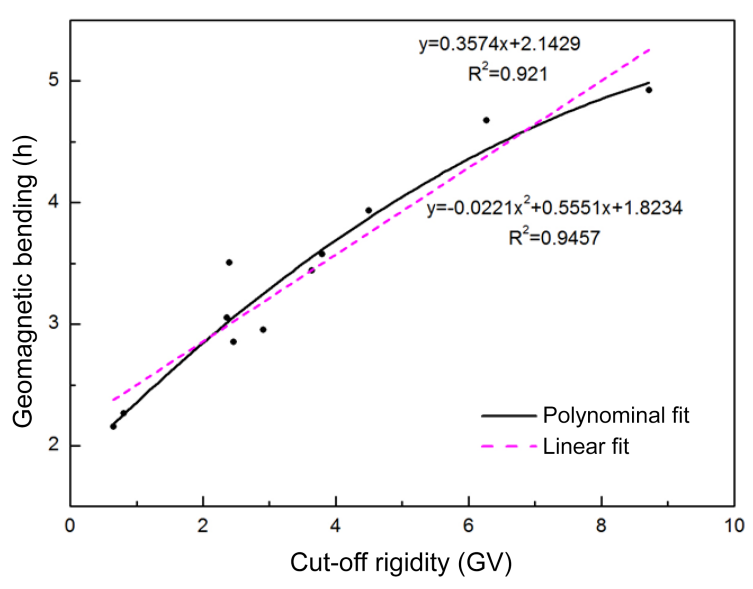

Figure 4. Diagram of the geomagnetic bending of all the stations with respect to their geomagnetic threshold rigidity.

field along the parallel of latitude presents exactly the same effect (ignoring local micro-anomalies). Therefore, it is expected that the diurnal phase (time of maximum) will be at the same direction for the NMs of GR 1, GR 2, and GR 3 groups, while there will be a shift for the diurnal vectors of GR 4 and GR 5.

The results were compared to previous calculations by Hatton and Carswell (1963), McCracken et al. (1968), Chaloupka et al. (1970), and Smart et al. (2000). It is observed that there are a few discrepancies between current and older calculations due to the evolution of the geomagnetic field through the years and the accuracy and the computing power nowadays. A certain improvement is the capability of having a greater detail in the asymptotic cones because of the increased number of points, which correspond to different energies of incoming particles.

The calculated magnetic bending of each station as a function of the cut-off rigidity using, at the same time, linear and second-order polynomial fits is presented in Fig. 4. From this work can be deduced that the relation between the magnetic bending and the corresponding cut-off rigidity of each station is almost linear since the second-order term is only $3.9 \%$ of the linear order. At the same time, a second-order polynomial fit is only $2.6 \%$ more accurate than the linear fit. This result indicates that there is an almost linear relation between the magnetic field and the geographic latitude of each station of the Northern Hemisphere.

\section{Diurnal anisotropy vectors}

\subsection{Diurnal anisotropy during the solar cycle}

From the normalized CR intensity recorded at the NMs used in this work over the period 2001-2014 (Fig. 2), we can clearly see CR variations related to the 11-year SC. During the year of solar maximum, 2001, the CR intensity reaches the lowest values with respect to the rest of the interval, while it increases towards the years 2008-2009 and records a maximum value during the same period as when the unusual minimum of solar activity between solar cycles 23 and 24 occurred. During the years 2010-2014 we observe a decreasing cosmic ray intensity corresponding to the minimaximum of solar cycle 24 with a lower value in the solar maximum of this cycle during the years 2012 and 2014 (Aslam and Badruddin, 2015). The years 2003 and 2005 are distinguished from the others due to their strong solar and CR events (Tezari and Mavromichalaki, 2016), even though the applied filters eliminate specific days. Days without events exhibiting intense activity below the filter threshold are clearly still included in the analysis.

An analogous behavior is observed in the time profiles of the diurnal amplitude and the time of maximum in LT and UT for the years 2001-2014 illustrated in Fig. 5. The examined time period covers the maximum and the descending phase of SC 23 (2001-2007) the deep minimum between SCs 23 and 24 (2008-2009) and the ascending phase and the maximum of SC 24 (2010-2014). It is observed that the annual diurnal amplitude varies according to the 11-year SC, while there is not a similar behavior for the diurnal phase, which is supposed to vary within a period of 22 years (one magnetic SC). This is consistent with the results of Bieber and Chen (1991), Singh and Badruddin (2006), Kudela et al. (2008a), Mishra and Mishra (2008), Tiwari et al. (2012), and Tezari and Mavromichalaki (2016).

The annual average diurnal amplitude is maximum during the declining phase of SC 23 and remains stable during the minima period of solar activity (2007-2009), while it obtains its minimum value during 2009 (Mailyan and Chilingarian, 2010). Then, the annual average diurnal amplitude increases again during the ascending phase of SC 24. Consequently, the amplitude of the diurnal anisotropy displays a clear 11year sunspot cycle variation, with minima occurring on or near sunspot minimum and maxima near sunspot maximums (Bieber and Chen, 1991; Tiwari et al., 2005, 2012; Sabbah, 2013). A small increase in diurnal amplitude is observed during 2005, due to various events, such as coronal mass ejections (CMEs) and magnetic storms. The amplitude is almost independent of the cut-off rigidity of the station (Bieber and Chen, 1991; Singh and Badruddin, 2006), while the amplitude variation is greater for higher-latitude stations in comparison to middle-latitude stations, such as Athens (Mailyan and Chilingarian, 2010).

As mentioned, the phase of the maximum does not have a clear correlation with the SC. The phase minimum appears to be at the minimum of solar activity (2009). It is proposed that the phase variation has two components: the 22-year and the 11-year components. The first component is dominant at high-latitude NMs and is controlled by drift effects, while both of them are important for middle- and low-latitude stations. This is consistent with the results of Bieber and Chen (1991), Oh et al. (2010), and Sabbah (2013) at lower 


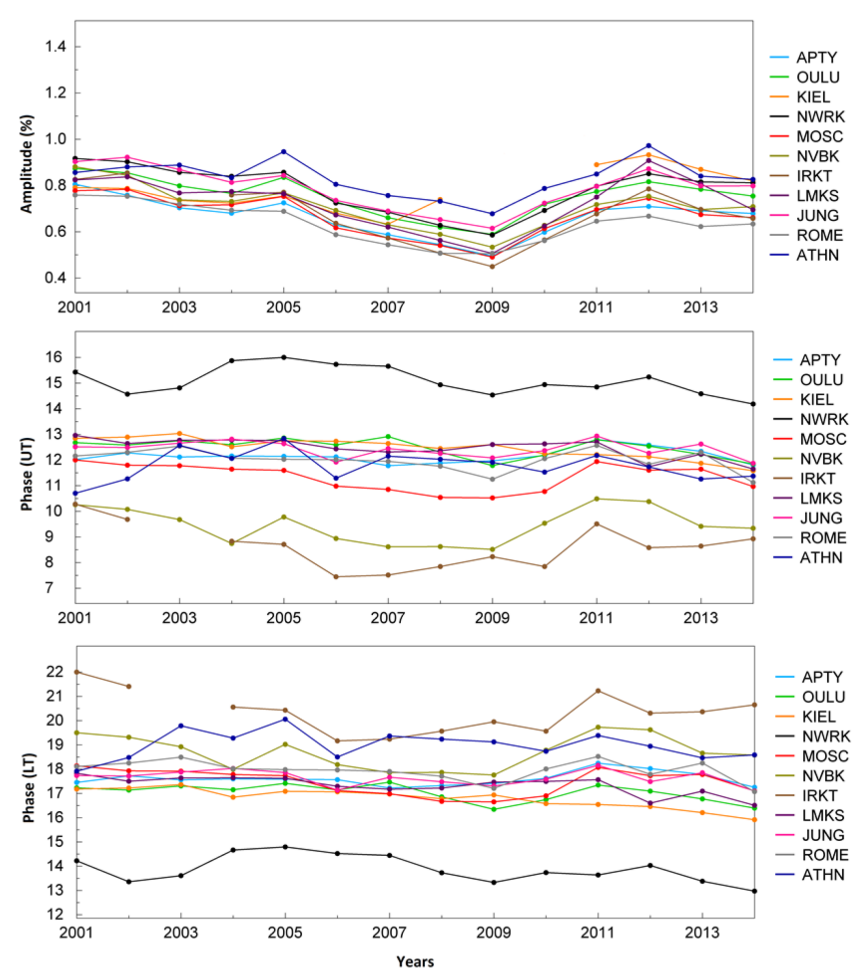

Figure 5. Time profiles of the diurnal amplitude (upper panel) and the time of maximum in UT and LT (middle and lower panels) of all the stations for the years 2001-2014.

energies, as well as with the recent results at higher cut-off rigidities and even at muon telescopes (Kudela and Sabbah, 2016). Athens shows the largest phase variation, as it has the highest threshold rigidity (Bieber and Chen, 1991). It is also observed that there is no significant phase shift during the descending phase of SC 23 and it remains invariant during the ascending phase of SC 24, which is in agreement with the "odd-even" SC model proposed by Tiwari et al. (2012).

It is known that the SMF field reverses at around the solar maximum activity (Ahluwalia, 1988). In this case the SMF reverses from positive to negative polarity in 2001 and from negative to positive in 2013. This confirms that the phase remains in the same polarity during the examined time period. Regarding solar cycle 23, the north polar reversal has been reported to be in February 2001 and the south polar reversal in September 2001 (Durrant and Wilson, 2003). According to another work, the north polar reversal of the Sun occurred in May 2001 and the south polar reversal in January 2002 (Bilenko, 2002). Gopalswamy et al. (2003) noted that the magnetic field strength had shown an "unsettled behavior" with several short-duration reversals during the time period 2000-2002. Regarding solar cycle 24, the polarity reversal of the solar magnetic field from negative to positive $(A>0)$ occurred during the year 2012 (Svalgaard and Kamide, 2013).

\subsection{Latitudinal and longitudinal distributions}

The annual diurnal vectors for all NMs used in this work are plotted on harmonic dial and are presented in Fig. 6 in LT and UT for the years 2001-2014. The diurnal vectors for the European NMs are across the Sun-Earth axis in UT, while they are shifted to earlier hours for the eastern stations (NVBK and IRKT) and to later hours for NWRK. Moreover, the European NMs seem to be consistent with the co-rotational model, as the diurnal anisotropy is in the $18 \mathrm{~h}$ direction (Krymsky, 1964; Rao, 1972) and they are aligned with the Parker spiral direction (Bieber and Chen, 1991). Once more, the stations NVBK, IRKT, and NWRK show a different behavior with NVBK and IRKT shifted to later hours and NWRK shifted to earlier hours, due to the geomagnetic bending correction. This behavior is probably due to the west-east asymmetry.

Due to the effect of the geomagnetic field on the daily variation in CR, the observed diurnal variation is dependent on the location of the NMs. Therefore, the observed values of the diurnal amplitude and phase vary quite significantly from one station to other, even though they are situated in the same latitude/longitude belt (Singh et al., 2013). In order to study the latitudinal and longitudinal distribution of CR intensity, the NMs used in this study are separated in five groups (see Sect. 2).

The term latitudinal distribution refers to the study of diurnal anisotropy for NMs that have almost the same geographic longitude but different geographic latitude. Therefore, they have different magnetic threshold rigidity, Rc. This is studied via using the annual diurnal vectors for GR 1 (Fig. 7a), GR 2 (Fig. 7b), and GR 3 (Fig. 7c). Plainaki et al. (2009) suggested that any differences in the counting rates of the ground level NMs of the same cut-off rigidity can be possibly attributed to the different asymptotic directions of viewing of these stations in relation to the location of the anisotropic solar particle flux source (2009). The diurnal vectors for the NMs of each of these groups points in almost the same direction, along the $18 \mathrm{~h}$, in agreement with the co-rotational model. It is observed that NMs of higher geographic latitude are shifted to earlier hours (OULU and KIEL), while NMs of middle latitude are shifted to later hours (ATHN and ROME). ATHN is shifted to later hours, probably due to the fact that it has the highest cut-off rigidity. Polar stations also exhibit the same behavior (GR 3).

The term longitudinal distribution refers to the study of diurnal anisotropy for NMs that have almost the same geographic latitude but different geographic longitude. Therefore, they have similar magnetic threshold rigidity Rc. The longitudinal diurnal anisotropy is studied by using the annual vectors for GR 4 (Fig. 7d) and GR 5 (Fig. 7e). It is observed that while we are moving from the western to eastern direction we are shifting to later hours. This is probably due to east-west asymmetry, which is greater in higher-latitude 

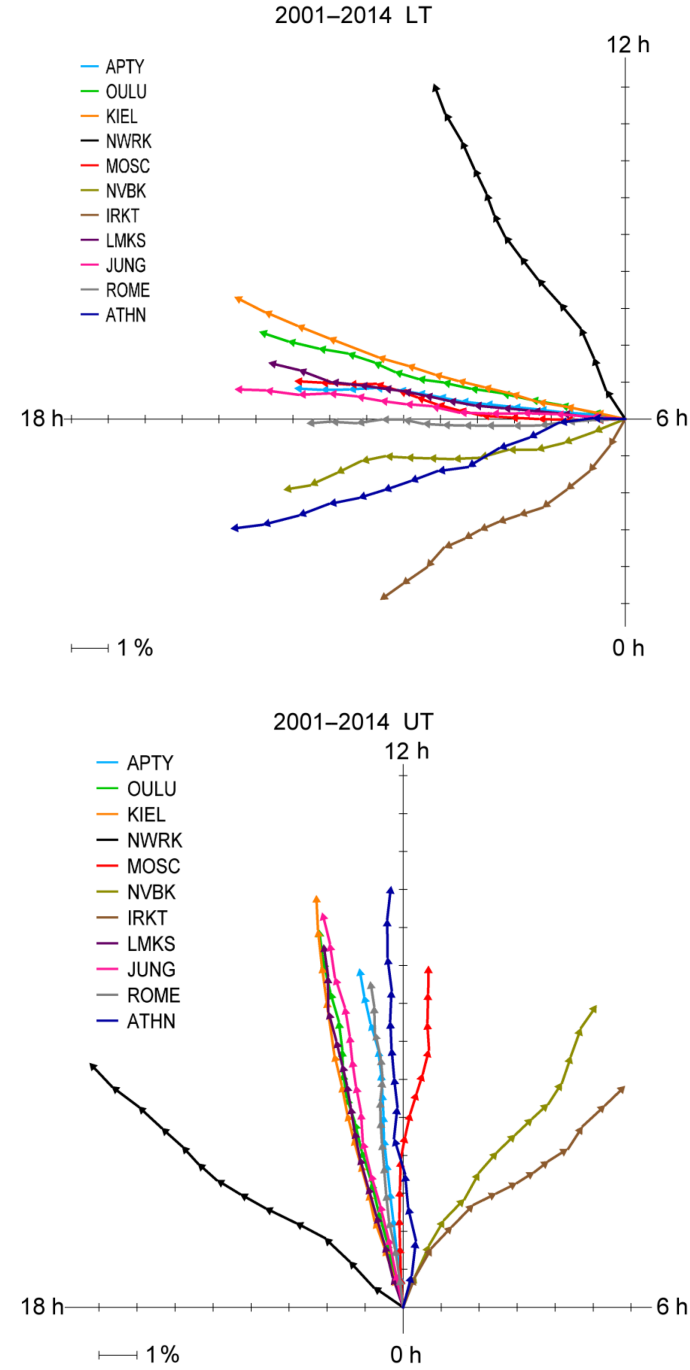

Figure 6. Annual diurnal anisotropy vectors for all NMs for the years 2001-2014 in LT (upper panel) and in UT (lower panel).

stations, over $50^{\circ} \mathrm{N}$ (Johnson, 1941; Burbury and Fenton, 1952).

\subsection{Diurnal anisotropy during strong cosmic ray events}

Solar and CR extreme events, such as GLEs, FDs, and MEs, strongly modulate the short-term diurnal anisotropy. As a result, the harmonic dials of the diurnal anisotropy exhibit very high ranges, as the NMs do not record the events with the same intensity due to their different cut-off rigidities and asymptotic cones (Plainaki et al., 2009; Tezari and Mavromichalaki, 2016). Typical examples of the daily diurnal vectors of selected extreme events during the studied period are illustrated in Figs. 8, 9, and 10, where it is observed that longitudinal and latitudinal diurnal anisotropy present a similar behavior.
In October 2003 (Fig. 8), the "Mother of Halos", a massive CME, took place on 28 October 2003, provoking GLE 65 and a series of FDs (Eroshenko et al., 2004). The GLE event was recorded by all NMs (Plainaki et al., 2005), mainly those of higher or polar latitude, as a huge enlargement of the diurnal amplitude, while the large FD in the same period appeared as a strong reversal of the diurnal vectors with a shift to earlier hours, except for the stations APTY and OULU, where a shift to later hours was observed. The different behavior of these stations is probably due to the fact that they are located in polar regions and have by far low cut-off rigidity. The NMs of GR 4 and GR 5 exhibit exactly the same variation, with a phase shift to earlier hours as we move towards the east, as was expected from the distribution of the asymptotic cones of this NMs group.

In November 2003 (Fig. 9), during the magnetospheric event of 20 November 2003, a great enlargement of the diurnal amplitude was recorded by ATHN station and aurora was visible even from lower-latitudes stations, similar to the behavior of a GLE in northern stations. The variation in the amplitude was not recorded by the higher-latitude stations, but a great disturbance, in the form of reversals and loops of the diurnal vectors, is observed.

In May 2005 (Fig. 10), a FD took place in 11 May 2005 and was recorded with a reversal of the diurnal vectors and a shift to later hours, resulting in strong fluctuations and loops, likely due to the convective-diffusive mechanism (Mavromichalaki, 1989; Tezari and Mavromichalaki, 2016). This behavior is similar for all studied NMs. This FD was observed in all NMs at the same time and is classified as a simultaneous one by Lee et al. (2015). These kinds of FDs are not affected by the diurnal anisotropy, while the nonsimultaneous FDs are affected by the CR diurnal variation and their onset time is restricted to the daytime.

It is evident that stations located in a relatively high geographic latitude present the same behavior, while polar stations (e.g., APTY, OULU) or middle-latitude stations (e.g., ATHN) show deviations; however, it is observed that latitudinal and longitudinal distribution of diurnal anisotropy present a similar pattern during extreme events.

\section{Discussion and conclusions}

The solar modulation of GCR into the heliosphere induces the solar diurnal anisotropy (Parker, 1964; Forman and Gleeson, 1975). As CRs enter the heliosphere and gyrate along interplanetary magnetic field (IMF) line, various anomalies of the magnetic flux scatter them from their gyro-orbits, resulting in diffusion. At the same time the solar wind converts the CR outward, causing particles traveling along a regular portion of magnetic field lines to undergo magnetic curvature and drifts. The long-term studies of solar diurnal variation provide information about the average behavior of $\mathrm{CR}$ at the Earth's proximity and, consequently, about the diurnal 


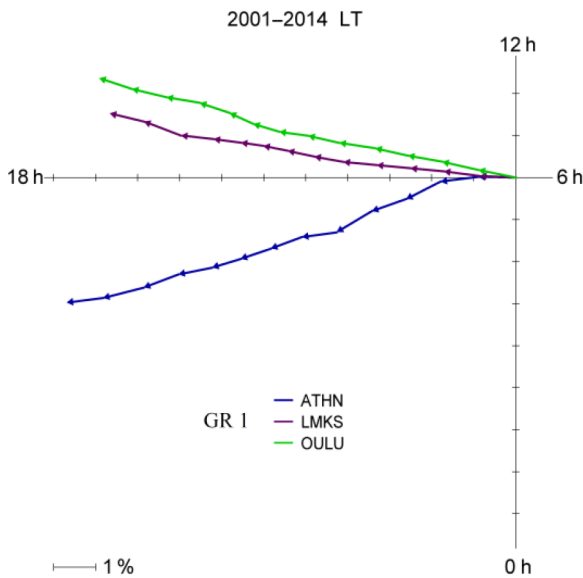

(a)

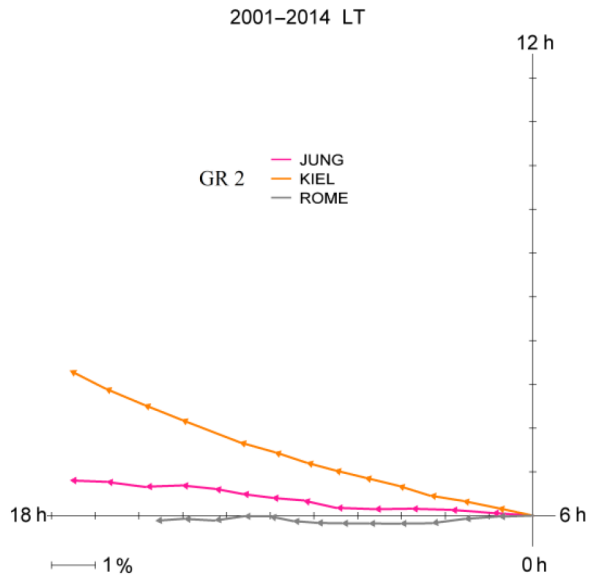

(b)

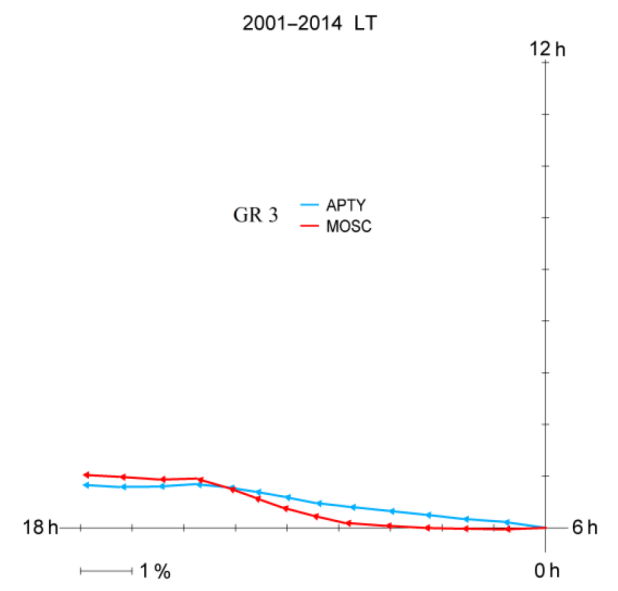

(c)

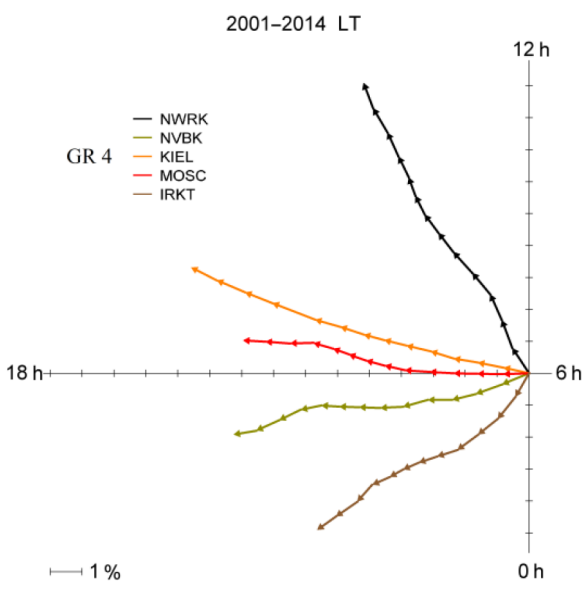

(d)

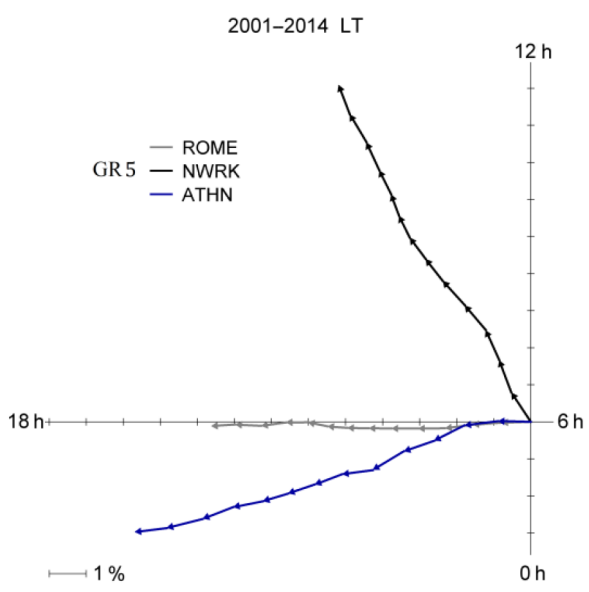

(e)

Figure 7. Annual diurnal vectors in LT (a) for GR 1 (ATHN, LMKS, and OULU), (b) for GR 2 (JUNG, KIEL, and ROME), (c) for GR 3 (APTY and MOSC), (d) for GR 4 (IRKT, KIEL, MOSC, NWRK, and NVBK), and (e) for GR 5 (ATHN, ROME, and NWRK). 


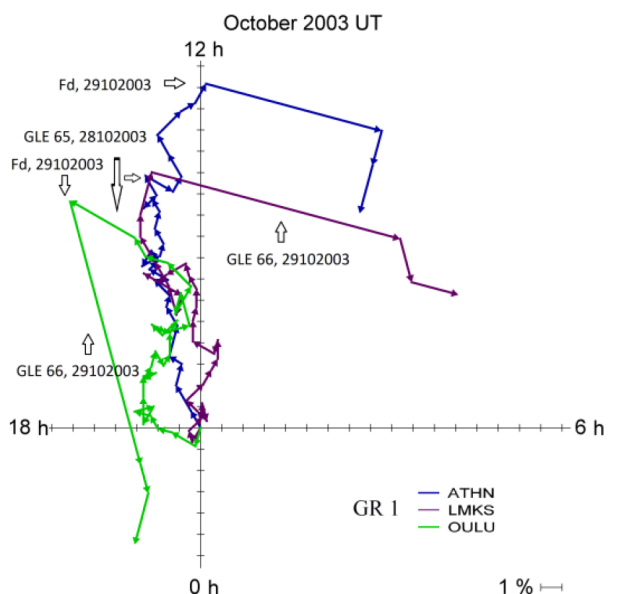

(a)

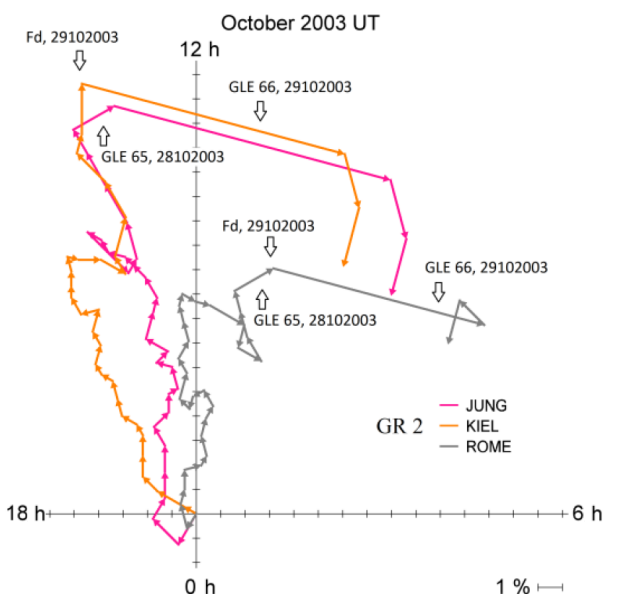

(b)

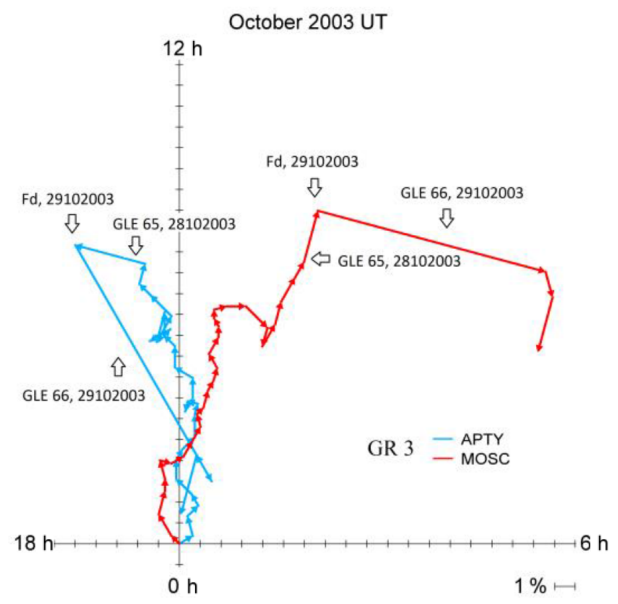

(c)

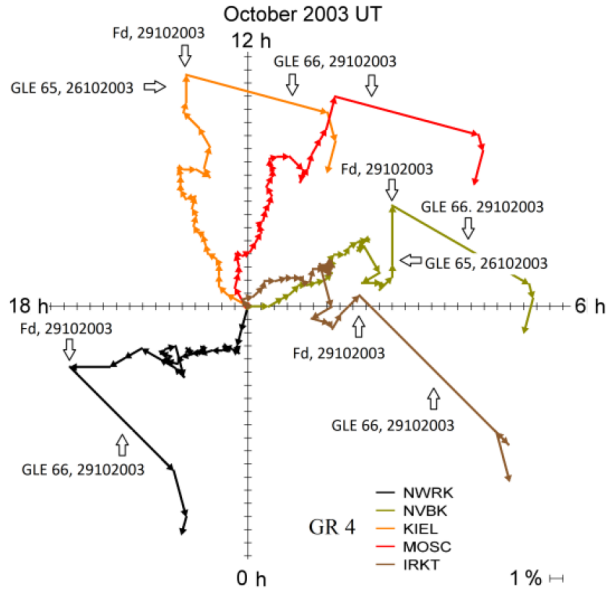

(d)

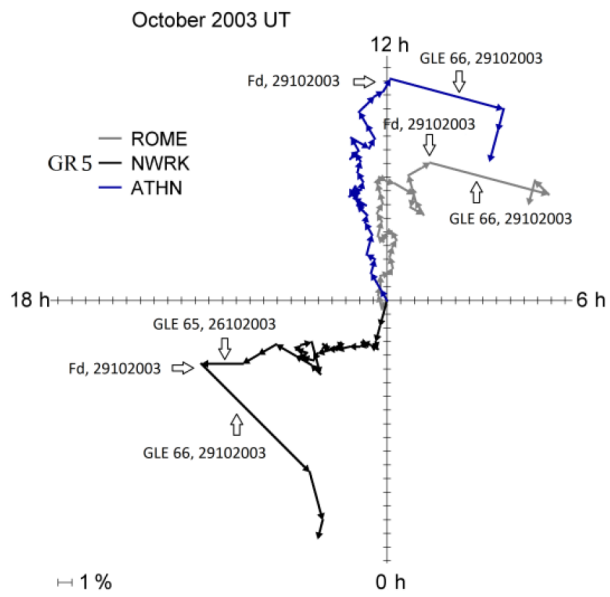

(e)

Figure 8. Daily diurnal vectors for all the groups of the stations for the month October 2003. 


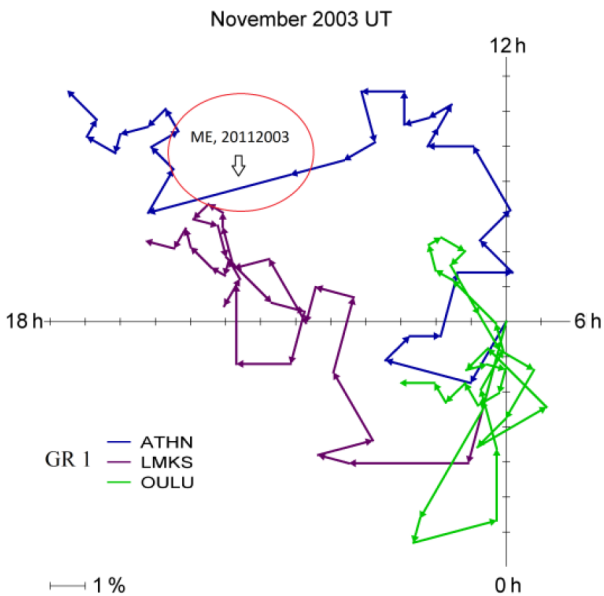

(a)

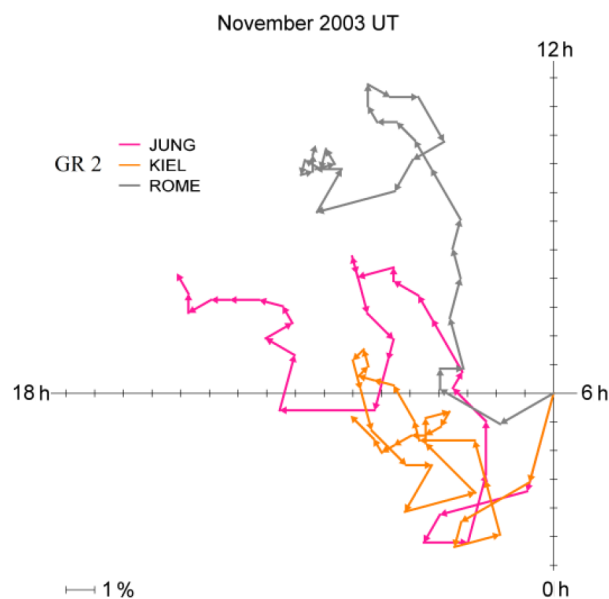

(b)

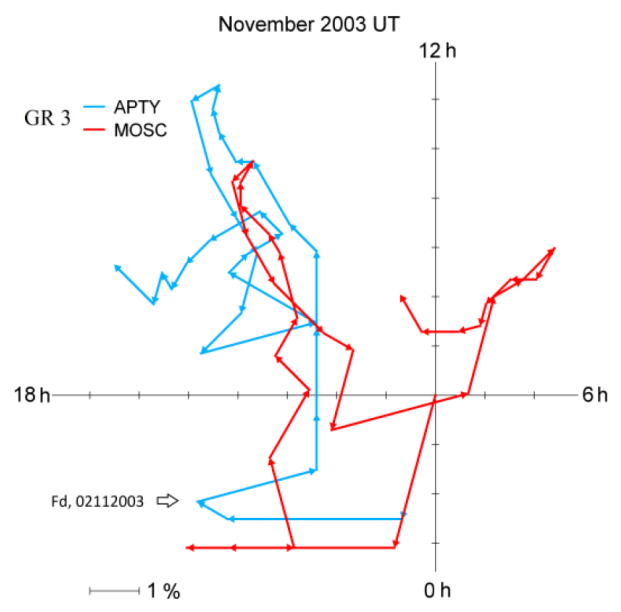

(c)

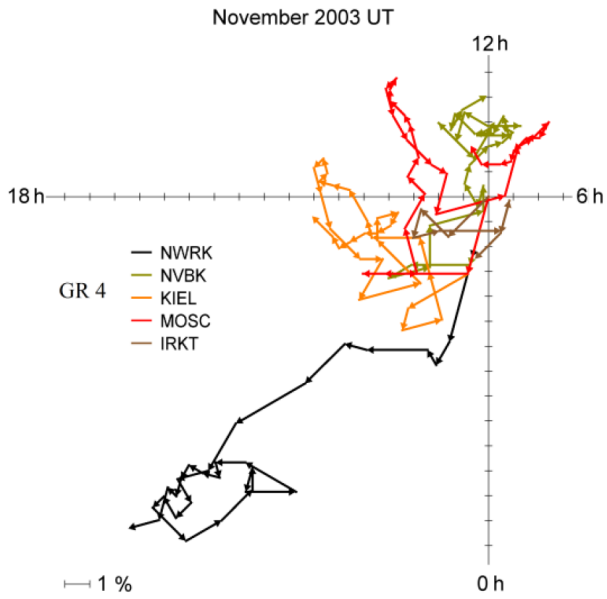

(d)

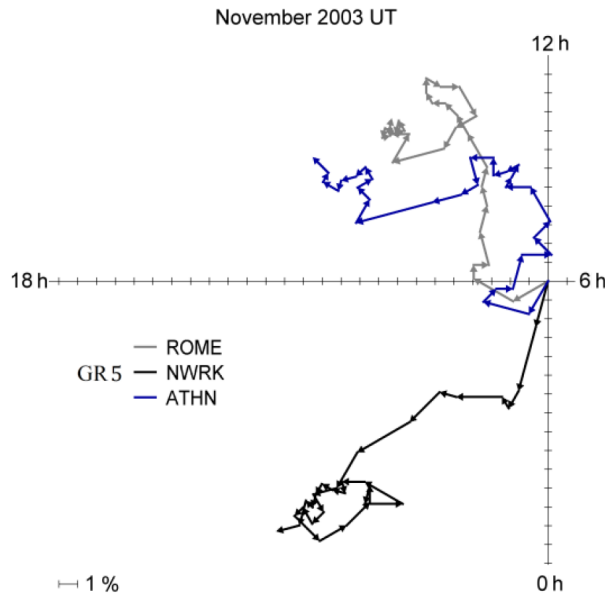

(e)

Figure 9. Daily diurnal vectors for all the groups of the stations for the month November 2003. 


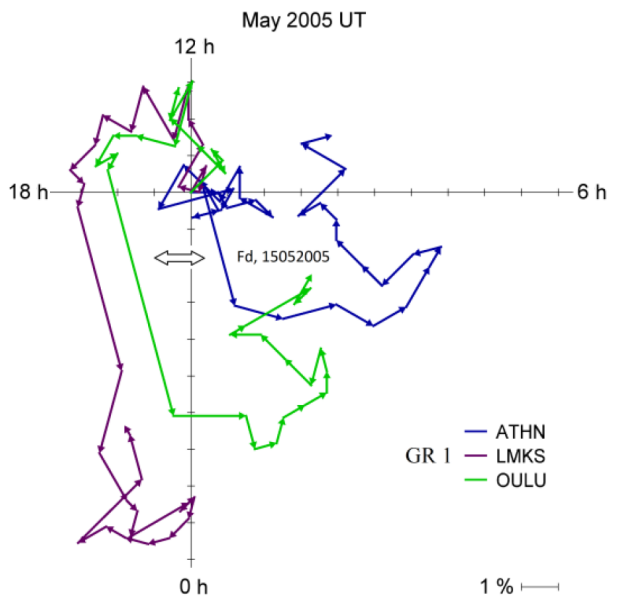

(a)

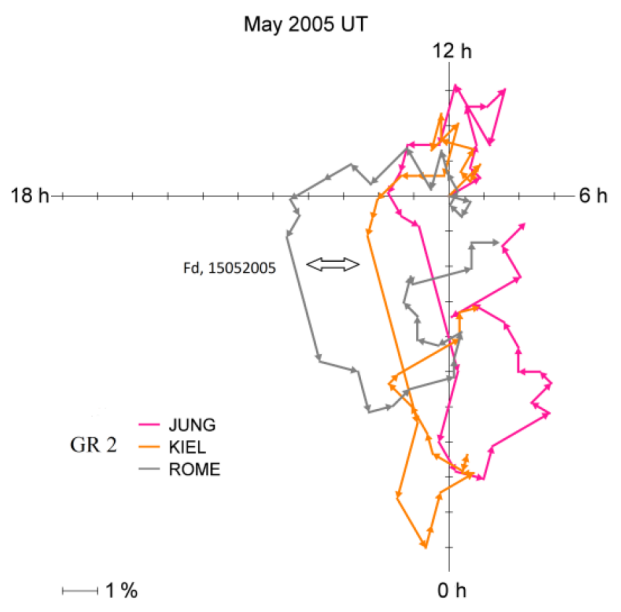

(b)

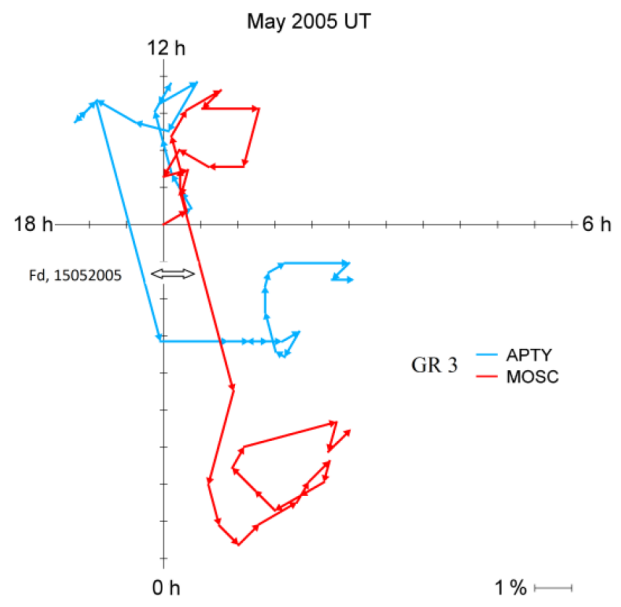

(c)

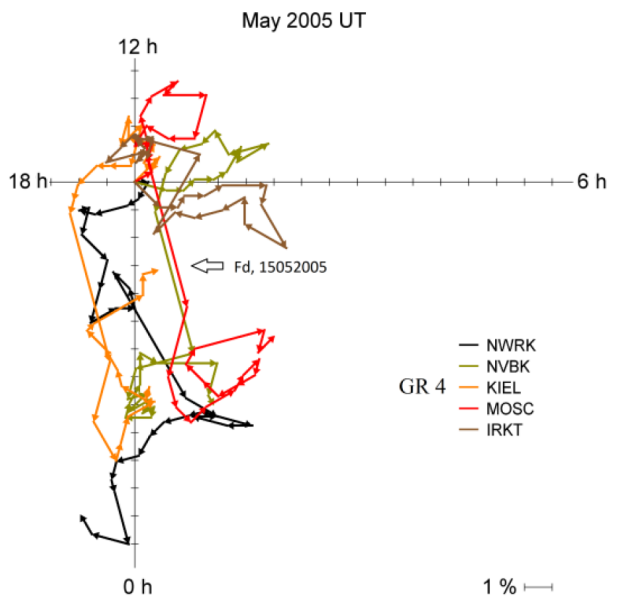

(d)

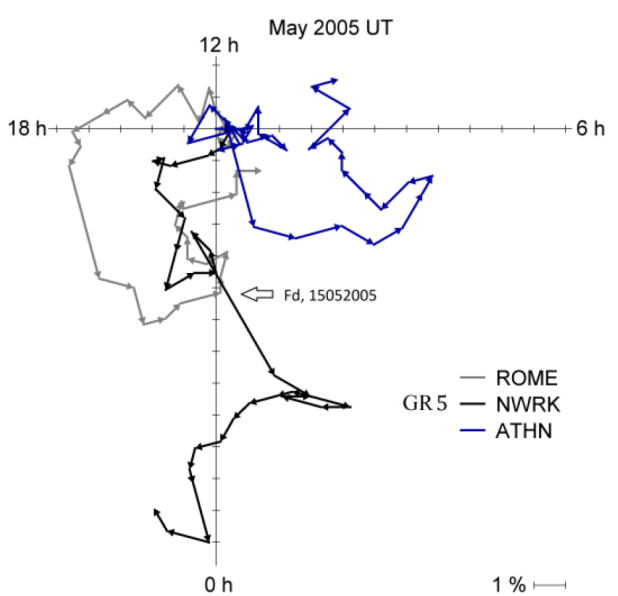

(e)

Figure 10. Daily diurnal vectors for all the groups of the stations for the month May 2005. 
anisotropy observed by ground-based monitors. This information may be useful for better understanding of the modulation processes (Venkatesan and Badruddin, 1990; Hall et al., 1996).

The asymptotic directions of viewing of the ground-based NMs are determined by the configuration of the magnetosphere and so they are sensitive to the time of day, to the time of year, and to the level of the magnetic disturbances. The distortion of the geomagnetic field by the solar wind may cause a variation in the viewing direction at a specified energy according to the local daytime. The diurnal amplitude is affected by the asymptotic latitude of primary CR arrival, while the diurnal phase is influenced by the asymptotic longitude for a specific solar diurnal variation (Humble and Duldig, 2003). Therefore, the study of asymptotic cones of viewing is necessary in the analysis of diurnal anisotropy. Other effects, such as sidereal variations, are considered negligible for long-term studies.

In this work, the characteristics of the CR diurnal anisotropy for 11 NMs during the years 2001 to 2014; covering a complete SC, are studied. As the diurnal anisotropy is a phenomenon of local time, the study of the asymptotic cones of acceptance of the NMs is very important for the determination of the correction due to the geomagnetic bending. A detailed study of both longitudinal and latitudinal distribution of the diurnal anisotropy is performed for the first time and supports the idea that the behavior of the diurnal anisotropy of CR intensity is determined not by the magnetic threshold rigidity but actually by a combination of this and the geographic coordinates of the given NM station.

The main results of this study can be summarized as follows:

- The use of the geomagnetic bending and the updated geomagnetic field models (with external current systems such as Tsyganenko96), along with the developed software, leads to a better description of the diurnal characteristics for various geographic positions of NMs during the examined years 2001-2014, as well as for selected transitional events in the inner heliosphere.

- In order to have more accurate estimations of the diurnal anisotropy characteristics, the asymptotic cones of viewing for the examined NMs have been taken into account and the geomagnetic bending for each station has been calculated (Table 1) (Chaloupka et al., 1970; Humble and Duldig, 2003)

- It was shown that the asymptotic cones of acceptance are almost identical for each group of the latitudinal distribution (GR 1, GR 2, GR 3), supporting the fact that the diurnal phase leads to the same direction. On the other hand, the asymptotic cones of the NMs of the longitudinal distribution (GR 4) are shifted in parallel, resulting in a shift of the time of maximum.
- The annual average diurnal amplitude of the cosmic ray recordings examined in this work follows the 11-year variation in the $\mathrm{SC}$ well, presenting a maximum value during the maximum periods of solar cycles 23 and 24 (years 2001-2003 and 2012 respectively) and a minimum value during the solar minimum between the cycles 23 and 24 (year 2009). On the other hand, the diurnal phase does not seem to present an analogous behavior that is possibly extended to the 22-year magnetic cycle (Bieber and Chen, 1991; Tiwari et al., 2012).

- During the examined period the diurnal anisotropy amplitude of cosmic rays is almost identical for all NMs, while its variation is greater for the NMs located in high and polar geographic latitudes in comparison to the middle- and equatorial-latitude stations (Table 2).

- We found a phase shift of the time of maximum to later hours during the examined time period in the stations with increasing cut-off rigidity with the greatest one observed in the Athens NM (cut-off rigidity 8.53 GV).

- It is concluded that the asymptotic latitude and the asymptotic longitude of the primary $\mathrm{CR}$ arrival affect the diurnal amplitude and the diurnal time of maximum, respectively, for a given solar diurnal anisotropy (Humble and Duldig, 2003). The variations in asymptotic directions during the day do not have a great influence on the diurnal variation observed at the NMs, as the solar diurnal anisotropy is weakly rigidity-dependent.

- There is no evidence for a systematic phase shift of the yearly time of maximum of the diurnal anisotropy of all stations for the examined period. This is because the period under study is characterized by solar magnetic field of the same polarity $(A<0)$ (Gopalswamy, 2003).

- Our results on the yearly average diurnal time of maximum for all the NMs are consistent with the corotational model, which sets the value of this parameter at around $18 \mathrm{~h}$ in LT (Parker, 1964). We found that the estimations corresponding to NVBK and IRKT stations are shifted to later hours in LT, while the one corresponding to NWRK is shifted to earlier hours (Fig. 7d). The opposite behavior is observed in the case of the phase in UT. This different behavior of eastern and western NMs is probably due to the west-east asymmetry, which is evident for geographic latitudes greater than $50^{\circ} \mathrm{N}$ (Burbury and Fenton, 1952).

- Diurnal variation is an effect of convection and diffusion due to the steady outer GCR source, while it is also due to transitional effects in the inner heliosphere. Therefore, the diurnal anisotropy of cosmic rays is also affected on a short-term basis by intense cosmic ray events, such as GLEs, FDs, and MEs, which are modulated from the solar activity (Tezari and Mavromichalaki, 2016). 
In summary, a new software application, called the DIurnal Anisotropy Suite (DIAS) is introduced for the study of the characteristics and the representation of the diurnal anisotropy vectors. The online function of DIAS, hopefully available in the next years, will provide continuous diurnal variation monitoring. The related results will be useful for long- term space weather monitoring integrating current estimations based on ground-based and satellite data and/or modeling.

\section{Data availability}

Data can be found on the NMDB website (http://nmdb.eu) (NMDB, 2008). Additionally, many of the stations used in this work provide their data online on their own websites.

Acknowledgements. The authors are grateful to our colleagues of the Neutron Monitor Stations used in this work for kindly providing their CR data. We acknowledge the Neutron Monitor Database (www.nmdb.eu), founded under the European Union's Seventh Framework Programme (contract no. 213007), for providing CR data. Athens Neutron Monitor Station is supported by the Special Research Account of Athens University (70/4/5803). Thanks are due to K. Kudela of the Slovak Academy of Sciences for his important comments reviewing this work, M. A. Shea, and D. F. Smart of the USA Air Force Research Laboratory and E. Eroshenko and V. Yanke of the Russian Academy of Sciences for useful discussions. The authors express many thanks to the EGU 2015 General Assembly Scientific Committee, where part of this work was presented and A. Tezari received an Outstanding Student Poster Award.

The topical editor, M. Temmer, thanks the two anonymous referees for help in evaluating this paper.

\section{References}

Ahluwalia, H. S.: The regimes of the east- west and the radial anisotropies of cosmic rays in the heliosphere, Planet. Space Sci., 36, 1451-1459, doi:10.1016/0032-0633(88)90010-4, 1988.

Aslam, O. P. M. and Badruddin: Study of the cosmic ray modulation during the recent unusual minimum and the mini-maximum of solar cycle 24, Sol. Phys., 290, 2333-2353, doi:10.1007/s11207015-0753-5, 2015.

Belov, A., Eroshenko, E., Mavromichalaki, H., Plainaki, C., and Yanke, V.: Solar cosmic rays during the extremely high ground level enhancement on 23 February 1956, Ann. Geophys., 23, 2281-2291, doi:10.5194/angeo-23-2281-2005, 2005.

Bieber, J. W. and Chen, J. L.: Cosmic ray diurnal anisotropy, 193688: Implications for drift and modulation theories, Astrophys. J., 372, 301-313, doi:10.1086/169976, 1991.

Bilenko, I. A.: Coronal holes and the solar polar field reversal, Astron. Astrophys., 396, 657-666, doi:10.1051/00046361:20021412, 2002.

Burbury, D. W. P. and Fenton, K. B.: The High Latitude East-West Asymmetry of Cosmic Rays, Australian J. Scient. Res. A, 5, 47$58,1952$.
Burlaga, L. F. and Ness, N. F.: Voyager Observations of the Magnetic Field in the Distant Heliosphere, Space Science Rev., 83, 105-121, doi:10.1023/A:1005025613036, 1998.

Chaloupka, P., Dubinský, J., Fischer, S., and Kowalski, T.: Geomagnetic bending and effective angles of approach of the cosmic rays for various stations, Czech. J. Phys. B, 20, 447-452, doi:10.1007/BF01698403, 1970.

Desorgher, L., Kudela, K., Flückiger, E., Butikofer, R., Storini, M., and Kalegaev, V.: Comparison of Earth's magnetospheric magnetic field models in the context of cosmic ray physics, Acta Geophys., 57, 75-87, doi:10.2478/s11600-008-0065-3, 2009.

Dorman, L. I. and Fischer, S.: Diurnal cosmic ray variation with the inclusion of the geometrical effects and the asymptotic cones of approach, Can. J. Phys., 46, S809-S811, doi:10.1139/p68-356, 1968.

Durrant, C. J. and Wilson, P. R.: Observations and simulations of the polar field reversals in cycle 23, Sol. Phys., 214, 23-39, doi:10.1023/A:1024042918007, 2003.

Eroshenko, E., Belov, A., Mavromichalaki, H., Mariatos, G., Oleneva, V., Plainaki, C., and Yanke, V.: Cosmic ray variations during the two great bursts of solar activity in the 23rd solar cycle, Sol. Phys., 224, 345-358, 2004.

Firoz, K. A.: On cosmic ray diurnal variations: disturbed and quiet days, Proc. WDS'8 Part 2, 183-188, ISBN-13: 978-807378-066-1, 2008.

Firoz, K. A. and Kudela, K.: Cosmic Rays and Low Energy Particle Fluxes Proc. WDS‘8 Part 2, 106-110, ISBN-13: 978-80-7378024-1, 2007.

Forman, M. A. and Gleeson, L. F.: Cosmic ray streaming and anisotropies, Astrophys. Space Sci., 32, 77-94, doi:10.1007/BF00646218, 1975.

Gopalswamy, N., Lara, A., Yashiro, S., and Howard, A. R.: Coronal mass ejections and solar polarity reversal, Astrophys. J., 598, L63-L66, 2003.

Hall, D. L., Duldig, M. L., and Humble, J. E.: Analyses of sidereal and solar anisotropies of cosmic rays, Space Sci. Rev., 78, 401442, doi:10.1007/BF00171926, 1996.

Hatton, C. J. and Carswell, D. A.: Asymptotic directions of approach of vertically incident cosmic rays for 85 neutron monitor stations, Deep River Laboratory, Atomic Energy of Canada Limited-1824, Chalk River, Ontario, 1963.

Humble, J. E. and Duldig, M. L.: The Effect of Variable Directions of Viewing on the Interpretation of Diurnal Variations observed by Neutron Monitors, Proc. 28th ICRC 2003, 4197-4201, 2003.

Johnson, T. H.: The East-West Asymmetry of the cosmic radiation in high latitudes and the excess of positive mesotrons, Phys. Rev. Lett., 59, 11-15, doi:10.1103/PhysRev.59.11, 1941.

Krymsky, G. F.: Diffusion mechanism of diurnal cosmic ray variations, Geomagn. Aeronomy+, 4, 763-769, 1964.

Kudela, K., Langer, R., and Firoz, K.: On diurnal variation of cosmic rays: statistical study of neutron monitor data including Lomnický štít, Proc. 21st ECRS, Kosice, 4.15, 374-378, 2008a.

Kudela, K., Bučik, R., and Bobík, P.: On transmissivity of low energy cosmic rays in disturbed magnetosphere, Adv. Space Res., 42, 1300-1306, doi:10.1016/j.asr.2007.09.033, 2008b.

Kudela, K. and Sabbah, I.: Quasi-periodic variations of low energy cosmic rays, China Techn. Sci., 59, 547-557, 2016.

Lee, S., Oh, S., Yi, Y., Evenson, P., Jee, G., and Choi, H.: Longterm Statistical Analysis of the Simultaneity of Forbush Decrease 
Events at Middle Latitudes, J. Astron. Space Sci., 32, 33-38, doi:10.5140/JASS.2015.32.1.33, 2015.

Mailyan, B. and Chilingarian, A.: Investigation of diurnal variations of cosmic ray fluxes measured with using ASEC and NMDB monitors, Adv. Space Res., 45, 1380-1387, doi:10.1016/j.asr.2010.01.027, 2010.

Mavromichalaki, H.: Application of diffusion-convection model to diurnal anisotropy data, Earth Moon Planets, 47, 61-72, doi:10.1007/BF00056331, 1989.

Mavromichalaki, H., Papageorgiou, C., and Gerontidiou, M.: Solar cycle and 27-day variations of the diurnal anisotropy of cosmic rays during the solar cycle 24, Astrophys. Space Sci., 361, 69-77, doi:10.1007/s10509-016-2661-z, 2016.

McCracken, K. G., Rao, U. R., Fowler, B. C., Shea, M. A., and Smart, D. F.: Cosmic Ray Tables (Asymptotic Directions, Variational Coefficients and Cut-off Rigidities), Instruction Manual No. 10, Annals of the IQSY, 198-214, MIT Press, Cambridge, 1968.

Mishra, R. K. and Mishra, R. A.: Cosmic ray diurnal anisotropy related to solar activity, Turkish J. Phys., 29, 55-61, 2005.

Mishra, R. K. and Mishra, R. A.: Cosmic ray daily variation and solar activity on anomalous days, Rom. J. Phys., 53, 925-932, 2008.

NMDB (Neutron Monitor Database): Real-time database for high resolution neutron monitor measurements, available at: http:// www.nmdb.eu/ (last access: 5 May 2016), 2008.

Oh, S. Y., Yi, Y., and Bieber, J. W.: Modulation cycles of galactic cosmic ray diurnal anisotropy variation, Sol. Phys., 262, 199212, doi:10.1007/s11207-009-9504-9, 2010.

Parker, E. N.: Theory of streaming of cosmic rays and the diurnal variation, Planet. Space Sci., 12, 735-749, doi:10.1016/00320633(64)90054-6, 1964.

Plainaki, C., Belov, A., Eroshenko, E., Kurt, V., Mavromichalaki, H., and Yanke, V.: Unexpected burst of solar activity recorded by neutron monitors during October-November 2003, Adv. Space Res., 35, 691-696, 2005.

Plainaki, C., Belov, A., Eroshenko, E., Mavromichalaki, H., and Yanke, V.: Modeling ground level enhancements: event of 20 January 2005, J. Geophys. Res., 112, 4102, doi:10.1029/2006JA011926, 2007.

Plainaki, C., Mavromichalaki, H., Belov, A., Eroshenko, E., and Yanke, V.: Neutron monitor asymptotic directions of viewing during the event of 13 December 2006, Adv. Space Res., 43, 518-522, doi:10.1016/j.asr.2008.09.007, 2009.

Plainaki, C., Mavromichalaki, H., Laurenza, M., Gerontidou, M., Kanellakopoulos, A., and Storini, M.: The ground-level enhancement of 2012 may 17: derivation of solar proton event properties through the application of the NMBANGLE PPOLA model, Astrophys. J., 785, 12 pp., doi:10.1088/0004-637X/785/2/160, 2014.

Pomerantz, M. A. and Duggal, S. P.: The cosmic ray solar diurnal anisotropy, Space Sci. Rev., 12, 75-130, doi:10.1007/BF00172130, 1971.

Rao, U. R.: Solar modulation of galactic cosmic radiation, Space Sci. Rev., 12, 719-809, doi:10.1007/BF00173071, 1972.

Rao, U. R., McCracken, K. G., and Venkatesan, D.: Asymptotic cones of acceptance and their use in the study of the daily variation of cosmic radiation, J. Geophys. Res., 345-369, doi:10.1029/JZ068i002p00345, 1963.
Razdan, H. and Summers, A. L.: Asymptotic cones of acceptance of cosmic ray neutron monitors in a geomagnetic field distorted by the solar wind, J. Geophys. Res., 70, 719-724, doi:10.1029/JZ070i003p00719, 1965.

Sabbah, I.: Solar magnetic polarity dependency of the cosmic ray diurnal variation, J. Geophys. Res., 118, 4739-4747, doi:10.1002/jgra.50431, 2013.

Singh, A., Dubey, D., Singh, R. P., and Tiwari, A. K.: Variations of upper cut-off rigidity of cosmic ray diurnal anisotropy, Intern. J. Innov. Res. in Science, Engineering and Technology, 2, ISSN: 2319-8753, 2013.

Singh, M. and Badruddin: Study of the cosmic ray diurnal anisotropy during different solar and magnetic conditions, Sol. Phys., 233, 291-317, doi:10.1007/s11207-006-2050-9, 2006.

Shea, M. A., Smart, D. F., and McCracken, K. G.: A Study of Vertical Cutoff Rigidities Using Sixth Degree Simulations of the Geomagnetic Field, J. Geophys. Res., 70, 4117-4130, 1965.

Smart, D. F., Shea, M. A., and Fluckiger, E. O.: Magnetospheric models and Trajectories Computations, Space Sci. Rev., 93, 305333, doi:10.1023/A:1026556831199, 2000.

Storini, M., Shea, M. A., Smart, D. F., and Cordaro, E. G.: Cutoff Variability for the Antarctic Laboratory for Cosmic Rays (LARC: 1955-1995), Proc. 26th ICRC, 7, 402-405, 1999.

Svalgaard, L. and Kamide, Y.: Asymmetric solar polar field reversals, Astrophys. J., 763, 23, doi:10.1088/0004-637X/763/1/23, 2013.

Tezari, A. and Mavromichalaki, H.: Diurnal anisotropy of cosmic rays during intensive solar activity for the time period 2001-2014, New Astronomy, 46, 78-84, doi:10.1016/j.newast.2015.12.008, 2016.

Tiwari, C. M., Tiwari, D. P., and Shrivastava, P. K.: Anomalous behavior of cosmic ray diurnal anisotropy during descending phase of the solar cycle-22, Curr. Sci. India, 88, 1275-1278, 2005.

Tiwari, A. K., Singh, A., and Agrawal, S. P.: Study of the diurnal variation of cosmic rays during different phases of solar activity, Sol. Phys., 279, 253-267, doi:10.1007/s11207-012-9962-3, 2012.

Tsyganenko, N. A.: A magnetospheric magnetic field model with a warped tail current sheet, Planet. Space Sci., 37, 5-20, doi:10.1016/0032-0633(89)90066-4, 1989.

Tsyganenko, N. A.: Modeling the Earth's magnetospheric magnetic field confined within a realistic magnetopause, J. Geophys. Res., 100, 5599-5612, doi:10.1029/94JA03193, 1995.

Tsyganenko, N. A. and Stern, D. P.: Modeling the global magnetic field of the large-scale Birkeland current systems, J. Geophys. Res., 101, 27187-27198, doi:10.1029/96JA02735, 1996.

Venkatesan, D. and Badruddin: Cosmic ray intensity variations in 3-Dimensional heliosphere, Space Sci. Rev., 52, 121-194, doi:10.1007/BF00704241, 1990.

Yeeram, T. and Saengdokmai, N.: Effects of the heliospheric current sheet on trains of enhanced diurnal variation in galactic cosmic rays, Sol. Phys., 290, 2311-2331, doi:10.1007/s11207-0150744-6, 2015. 